\title{
Tattoo Removal by Surgery in a Bloodless Field
}

\author{
Maj J B Hull \\ FRCS(Ed), RAMC \\ Specialist in Surgery \\ Maj N B Corner \\ FRCS, RAMC \\ Senior Specialist in Surgery \\ Lt Col P Roberts \\ MBE, FRCS, MS, RAMC \\ Consultant Surgeon
}

\author{
Wg Cdr A I Attwood \\ FRCS, RAF \\ Consultant Plastic Surgeon \\ Queen Elizabeth Military Hospital, Stadium Road, Woolwich, London SE18 4QH
}

SUMMARY: Tattoo removal from the forearm often requires excision and split skin grafting. The authors propose the use of a bloodless field to facilitate accurate dermal peeling, to ensure complete removal of the pigment, and to reduce $O$ operative time.

\section{Introduction}

Removal of decorative tattoos is a commonly requested operation in the Armed Services. Many service men and women regret having had their skin indelibly marked when young, and approach the surgeon seeking removal. Many methods of tattoo ablation have been tried(1) but excision with or without split skin grafting, is still the most common method employed. Excision and closure of small linear tattoos is a simple matter and providing the surrounding skin can be mobilised without tension, the results are good. Large tattoos, usually on the forearm and hands, require skin grafting after excision. The best results are achieved if the very thinnest layer of skin is removed leaving the deep dermis intact(2). This dermal peel technique is complicated by a continuous ooze of blood from the dermis which obscures the operative field making identification of the correct plane of excision difficult. It is to overcome this difficulty that the use of an arterial tourniquet to produce a bloodless field is proposed.

\section{Method}

A pneumatic arterial tourniquet is applied to the upper arm and after exsanguination of the limb, the cuff is inflated to $100 \mathrm{~mm} \mathrm{Hg}$ above arterial blood pressure(3).

A skin marker pen is used to outline the tattoo but the exact contours of the tattoo are not closely followed in order that a smoothly rounded area is removed. The margin is incised with a No. 15 scalpel blade taking care not to penetrate through to the subcutaneous layer. An attempt should be made to bevel the incision as this will create less of a step between the surrounding skin and the graft. Using a No. 10 blade, the epidermis and upp dermis are now peeled away from the intact deeg "white" layer of dermis (Fig 1). Because there is ifT bleeding, the exact level to which the pigment hos penetrated is easily identifiable. This may be comparit with tattoo excision without tourniquet, where bleedi readily obscures the operative field (Fig 2).

After removal of the tattoo, the intact dermis is checked for any stray ink spots which are simply excised $\stackrel{0}{8}$ with scissors. A thin (0.012in) split skin graft, harvested $\mathbb{\mathbb { Z }}$ from the upper arm or thigh, is meshed or perforated $\overrightarrow{\overrightarrow{0}}$ and applied to the dermal bed. It is fixed in position with 3 either Histoacryl glue or skin staples. The graft is dressed with Jelonet, wool and crepe and then the tourniquet is released. Dressings are removed at 5 days and providing healing is satisfactory, no further treatment is required, other than routine graft care of moisturising cream and compression with tubigrip for optimum cosmetic results. The donor site is treated according to normal practice.

\section{Results and Discussion}

The method described has been used to remove tattoos from forearms in fifty patients at the Queen Elizabeth Military Hospital, Woolwich. In all cases the $\frac{7}{0}$ graft 'take' at five days was in excess of $95 \%$ and no areas required further surgery or application of stored skin. In $N$ all cases the result was cosmetically acceptable to the $N$ patient. In two cases where bilateral forearm tattoos $\mathrm{N}$ were excised, the bloodless field technique was only $\omega$ used on one side. The resulting graft healing was 


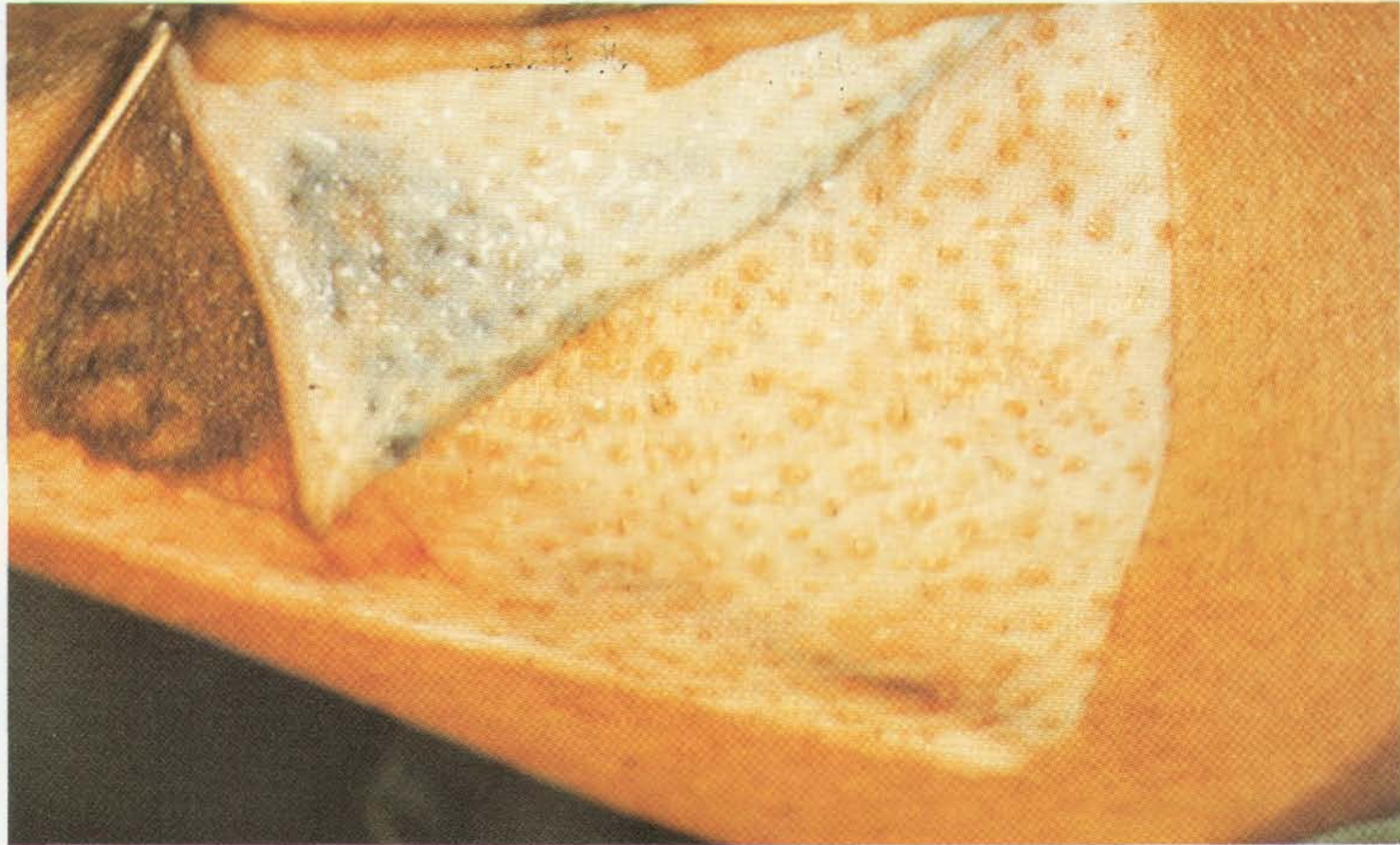

Fig 1. Dermal peel in a bloodless field.

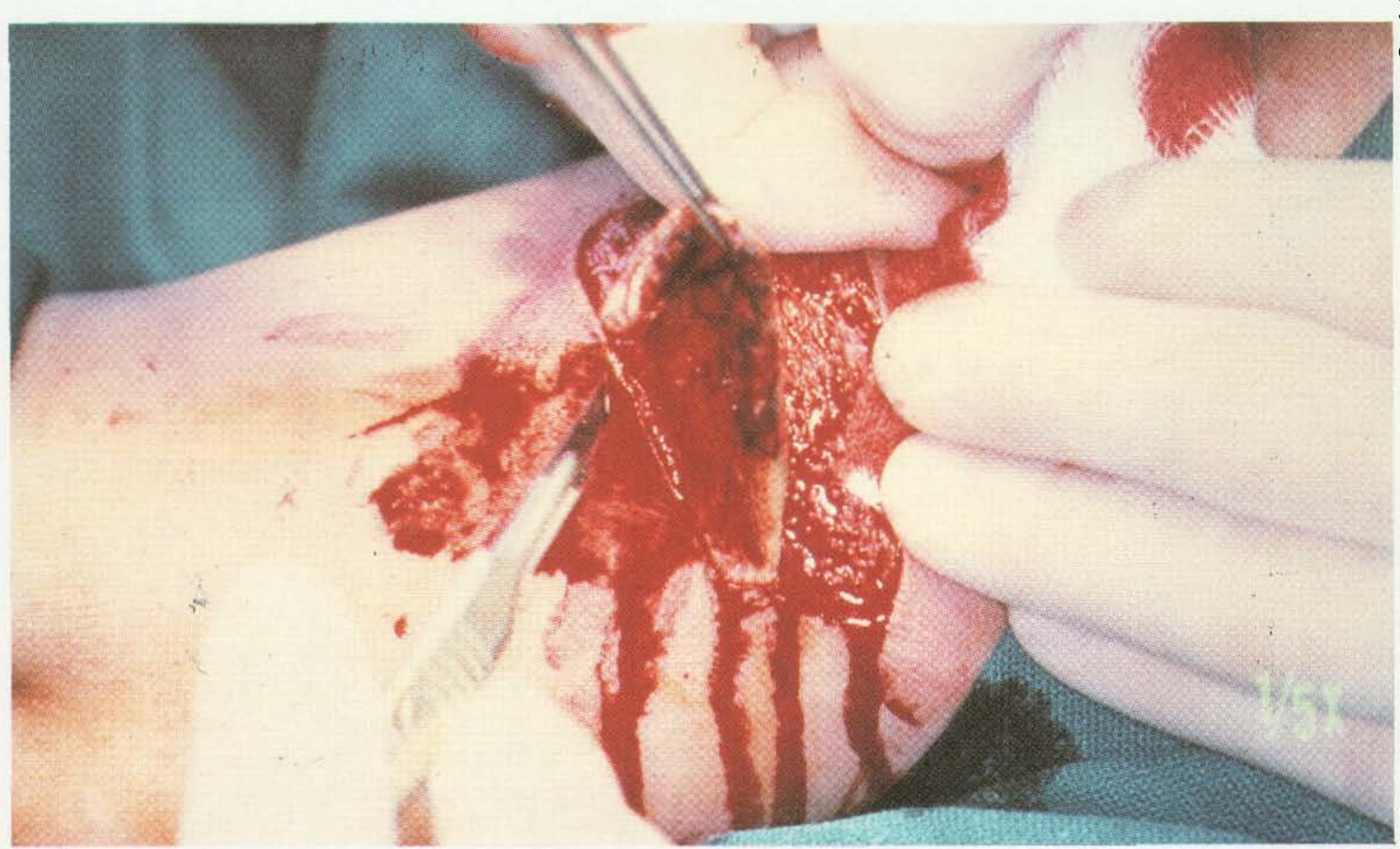

Fig 2. Tattoo removal without a tourniquet. 
compared at five days and the appearances of the two sides were indistinguishable.

This method is most suitable for removal of professional tattoos as the artist's pigment tends to penetrate to a more uniform depth allowing an even dermal peel. The ink penetration in amateur "homemade" tattoos is variable and is often found to be through to the subcutaneous tissues(4) making a dermal peel impossible.

It is important that the split skin graft is either perforated or meshed prior to its application to prevent the accumulation of blood under the graft after release of the tourniquet. If this is not undertaken, there is a very real risk of graft loss due to haematoma.

It is not suggested that use of this bloodless field technique markedly improves the end result after tattoo excision and grafting. It is clear, however, that even dermal peeling is made easier and quicker as bleeding is absent. It is also less likely that any stray ink spots will be inadvertently missed due to staining of the tissues with blood.

The method described is effective and safe with no complications or side effects. Tourniquet time is short and anaesthetic time is reduced. It is the authors' opinion that the use of a bloodless field should be recommended when either dermal peeling or serial shaving of a tattoo is undertaken.

\section{Acknowledgements}

The authors would like to thank Brigadier B C McDermott QHS, CBE, FRCS,L/RAMC for allowing this technique to be undertaken on his patients and $\mathrm{Mr}$ Peter Hesketh for producing the operative photographs.

\section{REFERENCES}

1. Arellano C, Rolendo J. Tattoo removal: Comparative study of six methods in the pig. J Plast Reconstr Surg 1983, 70: 699-703.

2. Vecchione $\mathbf{T} \mathbf{R}$. Tattoo removal using precise shave excision and dermal overgrafting. Ann Plast Surg 1988; 20: $443-447$.

3. Fletcher I R, Healey T E J. The arterial tourniquet. Ann $R$ Coll Surg Engl 1983; 65(6): 409-417.

4. Robinson J K. Tattoo removal. J Dermatol Surg Oncol 1985; 1: 14-16. 